\begin{tabular}{|c|l|}
\hline Title & Kinetic boundary condition in vapor-liquid two- phase sy stem during unsteady net evaporation/condensation \\
\hline Author(s) & Kon, Misaki; Kobay ashi, Kazumichi; W atanabe, Masao \\
\hline Citation & $\begin{array}{l}\text { European Journal of Mechanics- B/Fluids, 64, 81-92 } \\
\text { https://doi.org/40.1016/.euromechflu.2016.12.001 }\end{array}$ \\
\hline Issue Date & 2017-07 \\
\hline Doc URL & http://hdl.handle.net/2115/74844 \\
\hline Rights & $\begin{array}{l}\text { O 2017. This manuscript version is made available under the CC-BY-NC-ND 4.0 license } \\
\text { http://creativecommons.org/icenses/by-nc-nd/4.0/ }\end{array}$ \\
\hline Rights(URL) & http://creativecommons.org/icenses/by-nc-nd/4.0/ \\
\hline Type & article (author version) \\
\hline File Information & manuscript.pdf \\
\hline
\end{tabular}

Instructions for use 


\title{
Kinetic boundary condition in vapor-liquid two-phase system during unsteady net evaporation/condensation
}

\author{
Misaki Kon ${ }^{1, *}$, Kazumichi Kobayashi ${ }^{1}$, Masao Watanabe ${ }^{1}$ \\ Division of Mechanical and Space Engineering, Faculty of Engineering, Hokkaido University, Kita 13 Nishi \\ 8, Kita-ku, Sapporo, Hokkaido 060-8628, Japan
}

\begin{abstract}
Heat and mass transfer caused by nonequilibrium phase change (net evaporation/condensation) play a major role in a vapor-liquid two-phase flow. In general, liquid temperature changes with time because of the heat and mass transfer between the vapor and liquid phases; however, a precise investigation of the transport phenomena related to this temporal evolution of liquid temperature is still lacking. The aim of this study is to examine a kinetic boundary condition, which depends on liquid temperature, for the Boltzmann equation in a vaporliquid two-phase system with unsteady net evaporation/condensation. In this study, we confirmed whether the kinetic boundary condition follows the temporal evolution of liquid temperature attributed to unsteady net evaporation/condensation by using the molecular simulation based on mean-field kinetic theory, and then we validated the accuracy of the kinetic boundary condition by solving the initial boundary value problem of the Boltzmann equation in unsteady net evaporation/condensation. These results showed that the kinetic boundary condition follows the temporal evolution of liquid temperature in the simulation setting of this study. Furthermore, we concluded that the kinetic boundary condition that depends on liquid temperature is guaranteed to be accurate even in unsteady net evaporation/condensation by considering the temporal evolution of liquid temperature.
\end{abstract}

Keywords: kinetic boundary condition, evaporation and condensation, unsteady vapor-liquid two-phase flow, kinetic theory of gases

\section{Introduction}

As micro/nanofluidics progress, we should consider not only the rarefaction effect induced by the downsizing of a system but also nonequilibrium phase change (net evaporation/condensation) induced by molecular motion in the vicinity of the vapor-liquid

Email addresses: konmi@eng.hokudai.ac.jp (Misaki Kon ), kobakazu@eng.hokudai.ac.jp (Kazumichi Kobayashi), masao.watanabe@eng.hokudai.ac.jp (Masao Watanabe)

Preprint submitted to European Journal of Mechanics - B/Fluids

February 13, 2017 
interface[1]. Although net evaporation and condensation are essentially microscopic phenomena, it has been indicated that the heat and mass transfer attributed to net evaporation/condensation affects the dynamics of vapor-liquid two-phase flows, such as the motion of bubbles and droplets[2, 3, 4, 5, 6, 7]. In recent years, some applications using bubbles and droplets accompanied with net evaporation/condensation have been proposed; for instance, Ohl et al.,[8] and Kobayashi et al.,[9] suggested the base of medical technologies that use the shock wave and liquid jet produced by the collapse of the cavitation bubble, and Sazhin et al.,[10] proposed an optimal control technology for the diesel fuel by considering droplet evaporation. Hence, further investigation of vapor-liquid two-phase flow with net evaporation/condensation is required.

The most practical method to investigate a vapor-liquid two-phase flow is a numerical simulation of the fluid dynamic equations, especially one that utilizes the diffuse interface model or the Hertz-Knudsen-Langmuir model. However, there is a nonequilibrium transition layer between the bulk liquid and bulk vapor, which includes an interface layer and a Knudsen layer as shown in Fig. 1, in a micro-scale point of view. Note that the interface layer is synonymous with the term interphase, which has been used in the previous literature[11, 12, $13,14]$. The numerical simulation of the fluid dynamic equation with the diffuse interface model or the Hertz-Knudsen-Langmuir model would be advantageous if we would like to omit the complicated analysis in the nonequilibrium transition layer and obtain an overview of vapor-liquid two-phase flow with net evaporation/condensation. However, this numerical simulation cannot describe the certain transport phenomena in the nonequilibrium transition layer induced by the evaporation, reflection, and condensation of molecules, as shown in Fig. 1; therefore, it is essentially hard to quantitatively estimate vapor-liquid two-phase flows with net evaporation/condensation as have already pointed out $[4,15]$.

In this problem, an analytical approach based on molecular gas dynamics is essential. The Boltzmann equation, which governs molecular gas dynamics, describes the spatiotemporal development of the molecular velocity distribution function, $f$, in vapor which includes the Knudsen layer. However, the Boltzmann equation postulates a condition of the ideal gas. That means this equation cannot support the interface layer. Hence, we have to impose the velocity distribution function $f_{\text {out }}$, composed of molecules outgoing from the kinetic boundary into the Knudsen layer as the kinetic boundary condition (KBC), where the kinetic boundary refers to the boundary between the interface layer and Knudsen layer (see Fig. 1). In the classical approach to the molecular gas dynamics, the conventional KBCs that are simplified so that they may be solved analytically and satisfy the fundamental physical requirements have been utilized $[16,17,18]$.

In contrast, with the development of high-performance computing from the late 1990s to the present, numerous studies have performed a molecular simulation of the full vapor-liquid two-phase system including the nonequilibrium transition layer in terms of constructing the $\mathrm{KBC}[11,12,13,14,19,20,21,22,23,24,25,26,27]$. Although it is clear that liquid temperature changes with time according to the heat and mass transfer caused by net evaporation/condensation, most of the studies mentioned above assume a steady or quasi-steady net evaporation/condensation because of the high computational cost of molecular simulation. In general, an unsteady molecular simulation requires an ensemble average to estimate 
the macroscopic variables; hence, the computational cost of the unsteady molecular simulation is exceedingly higher than a steady one. Soga[18] showed that the solution for unsteady net evaporation/condensation with the normalized Maxwellian evolution differs from that of quasi-steady net evaporation/condensation by analyzing the linearized Boltzmann equation. However, the detailed investigation based on the micro-scale point of view of the $\mathrm{KBC}$ in unsteady net evaporation/condensation has never been carried out.

The aim of this study is to investigate the KBC in the vapor-liquid two-phase system with unsteady net evaporation/condensation by a molecular simulation based on mean-field kinetic theory. This type of molecular simulation allows us to reduce the computational cost compared with the typically used molecular dynamics simulation [15, 24, 25, 26, 27, $28,29]$. In this study, we examine whether the KBC can be imposed by simply specifying liquid temperature even in unsteady net evaporation/condensation in order to extend the previous results that the $\mathrm{KBC}$, which is established in the vapor-liquid two-phase system with steady net evaporation/condensation[24, 27], depends only on liquid temperature (a detailed explanation of this $\mathrm{KBC}$ is given in Section 2.1). As illustrated in Fig. 1, the interface layer has a finite thickness; hence, a delay time arises from molecules traveling in this finite interface layer in unsteady net evaporation and condensation. Note that it is not obvious that the functional form of the $\mathrm{KBC}$ is the same as that in steady net evaporation and condensation.

First, we simulate the unsteady net evaporation and condensation problem in a system composed two liquid slabs at different temperatures by using the molecular simulation based on mean-field kinetic theory (Section 2.3). Second, we confirm whether the functional form of the KBC at each time is the same as that in steady net evaporation and condensation (Section 3.1), and then we estimate the temporal evolution of liquid temperature to establish the KBC, which depends on liquid temperature, in unsteady net evaporation/condensation (Section 3.2). Finally, we carry out a numerical simulation of the Boltzmann equation with the KBC that varies with the temporal evolution of liquid temperature estimated in Section 3.2 and then validate the accuracy of the KBC by comparing the macroscopic variables, namely, vapor velocity and temperature, at each time obtained from the numerical simulation of the Boltzmann equation and the molecular simulation based on mean-field kinetic theory (Section 3.3).

\section{Methods}

\subsection{Kinetic boundary condition}

In this section, we explain the $\mathrm{KBC}$ that is established in the vapor-liquid two-phase system with steady net evaporation/condensation[24, 27]. As the starting point, we examine a conventional $\mathrm{KBC}$ that is expressed as the linear combination of the diffuse reflection and complete condensation conditions [30, 31],

$$
f_{\text {out }}=\left[\alpha_{\mathrm{e}} \rho^{*}\left(T_{\mathrm{L}}\right)+\left(1-\alpha_{\mathrm{c}}\right) \sigma\right] \hat{f}, \quad \xi_{z}>0,
$$

where $\rho^{*}$ is the saturated vapor density, $\alpha_{\mathrm{e}}$ and $\alpha_{\mathrm{c}}$ are evaporation and condensation coefficients, respectively, $\xi_{z}$ is the molecular velocity in the direction normal to the kinetic 
boundary; $\xi_{z}>0$ indicates the direction of molecular velocity outgoing from the kinetic boundary into the Knudsen layer, and $\hat{f}$ is a normalized molecular velocity distribution function; the normalized Maxwellian distribution at liquid temperature $T_{\mathrm{L}}$,

$$
\hat{f}\left(T_{\mathrm{L}}\right)=\frac{1}{\left(2 \pi R T_{\mathrm{L}}\right)^{\frac{3}{2}}} \exp \left(-\frac{\xi_{i}^{2}}{2 R T_{\mathrm{L}}}\right)
$$

is conventionally assumed, where $R$ is the gas constant. Furthermore, $\sigma$ is a parameter that is related to a molecular velocity distribution function $f_{\text {coll }}$, composed of molecules colliding onto the liquid from the vapor phase $\left(\xi_{z}<0\right)$. This parameter is defined as

$$
\sigma\left(\frac{R T_{\mathrm{L}}}{2 \pi}\right)^{\frac{1}{2}}=-\int_{\xi_{z}<0} \xi_{z} f_{\text {coll }} \mathrm{d} \boldsymbol{\xi}=J_{\text {coll }},
$$

where $J_{\text {coll }}$ is the molecular mass flux composed of molecules colliding onto the kinetic boundary from the Knudsen layer and $\int_{\xi_{z}<0} \mathrm{~d} \boldsymbol{\xi}=\int_{-\infty}^{0} \int_{-\infty}^{\infty} \int_{-\infty}^{\infty} \mathrm{d} \xi_{x} \mathrm{~d} \xi_{y} \mathrm{~d} \xi_{z}$. $f_{\text {coll }}$ at each time is obtained by solving the initial boundary value problem of the Boltzmann equation[30].

In terms of the definitions of $\alpha_{\mathrm{e}}$ and $\alpha_{\mathrm{c}}$, some different models have been proposed[13, $19,20,21,23]$. We adopt the widely used definitions of $\alpha_{\mathrm{e}}$ and $\alpha_{\mathrm{c}}$ as follows[11, 21, 32]:

$$
\alpha_{\mathrm{e}}=\frac{J_{\text {evap }}}{J_{\text {out }}^{*}}, \quad \alpha_{\mathrm{c}}=\frac{J_{\text {cond }}}{J_{\text {coll }}},
$$

where $J$ indicates the molecular mass flux that is related to each molecular motions at the kinetic boundary (see Fig. 1); $J_{\text {evap }}$ and $J_{\text {cond }}$ are the molecular mass fluxes composed of evaporation and condensation molecules, and $J_{\text {out }}$ is the molecular mass flux composed of molecules outgoing from the kinetic boundary into the Knudsen layer. Furthermore, $J_{\text {out }}^{*}$ is $J_{\text {out }}$ at the vapor-liquid equilibrium, where $J_{\text {out }}^{*}=J_{\text {coll }}^{*}=\rho^{*}\left(R T_{\mathrm{L}} /(2 \pi)\right)^{\frac{1}{2}}$. The relations of each molecular mass flux at the kinetic boundary are as follows [24]:

$$
J_{\text {out }}=J_{\text {ref }}+J_{\text {evap }}, \quad J_{\text {coll }}=J_{\text {ref }}+J_{\text {cond }},
$$

where $J_{\text {ref }}$ is the molecular mass flux composed of reflection molecules.

In our recent studies[24, 25], we formulated $\alpha_{\mathrm{e}}$ and $\alpha_{\mathrm{c}}$ for hard-sphere molecules in steady net evaporation/condensation with the use of the net mass flux $\rho v_{z}$, at the kinetic boundary; $\rho v_{z}$ is obtained as the difference between $J_{\text {out }}$ and $J_{\text {coll }}$. The mass flux relation at the kinetic boundary with steady net evaporation is as follows:

$$
\frac{\rho v_{z}}{J_{\text {out }}^{*}}=\beta_{\text {ne }}\left(T_{\mathrm{L}}\right)\left(1-\frac{J_{\text {coll }}}{J_{\text {coll }}^{*}}\right)=\beta_{\text {ne }}\left(T_{\mathrm{L}}\right)\left(1-\frac{\sigma}{\rho^{*}}\right),
$$

and that with net condensation is as follows:

$$
\frac{\rho v_{z}}{J_{\text {out }}^{*}}=\beta_{\mathrm{nc}}\left(T_{\mathrm{L}}\right)\left(1-\frac{\sigma}{\rho^{*}}\right) .
$$


In our recent studies[24, 27], we confirmed that $\rho v_{z}$ is well described as a linear function of $\sigma / \rho^{*}$ and furthermore that $\beta_{\text {ne }}$ and $\beta_{\text {nc }}$ depend only on liquid temperature. When $J_{\text {coll }}=0$ in Eq. (6), $\alpha_{\mathrm{e}}$ can be obtained according to the concept of spontaneous evaporation[21] as

$$
\alpha_{\mathrm{e}}=\beta_{\mathrm{ne}}\left(T_{\mathrm{L}}\right) \text {. }
$$

Furthermore, $\alpha_{\mathrm{c}}$ in net evaporation can be formulated as

$$
\alpha_{\mathrm{c}}=\alpha_{\mathrm{e}}=\beta_{\mathrm{ne}}\left(T_{\mathrm{L}}\right)
$$

and that in net condensation can be formulated as

$$
\alpha_{\mathrm{c}}=\frac{\rho^{*}}{\sigma}\left(\beta_{\mathrm{ne}}\left(T_{\mathrm{L}}\right)-\beta_{\mathrm{nc}}\left(T_{\mathrm{L}}\right)\right)+\beta_{\mathrm{nc}}\left(T_{\mathrm{L}}\right) .
$$

Note that we assume that $\hat{f}$ is the normalized Maxwellian distribution at liquid temperature (Eq. (2)) in the above derivations. The derivations of Eqs. (9) and (10) can be found in the literature[24, 27].

We can obtain the KBC in net evaporation by substituting Eqs. (8) and (9) into Eq. (1):

$$
f_{\text {out }}=\left[\beta_{\text {ne }}\left(T_{\mathrm{L}}\right)\left(\rho^{*}-\sigma\right)+\sigma\right] \hat{f}\left(T_{\mathrm{L}}\right), \text { for } \xi_{z}>0 .
$$

In the same way, we can obtain the KBC in net condensation by substituting Eqs. (8) and (10) into Eq. (1):

$$
f_{\text {out }}=\left[\beta_{\text {nc }}\left(T_{\mathrm{L}}\right)\left(\rho^{*}-\sigma\right)+\sigma\right] \hat{f}\left(T_{\mathrm{L}}\right), \text { for } \xi_{z}>0 .
$$

As already mentioned above, because $f_{\text {coll }}$ is obtained at each time by solving the initial boundary value problem of the Boltzmann equation, $\sigma$ is also obtained from Eq. (3). It is the most significant advantage that $\beta_{\mathrm{ne}}, \beta_{\mathrm{nc}}$, and $\hat{f}\left(T_{\mathrm{L}}\right)$ in Eqs. (11) and (12) depend only on liquid temperature in steady net evaporation/condensation.

\subsection{Molecular simulation based on mean-field kinetic theory}

One practical equation based on mean-field kinetic theory is the Enskog-Vlasov equation[33, 34]. This equation is a kinetic equation that takes into account a self-consistent force field $F_{i}$ generated by the Sutherland potential $\phi(r)$,

$$
\phi(r)= \begin{cases}+\infty & (r<a) \\ -\phi_{a}\left(\frac{r}{a}\right)^{-\gamma} & (r \geq a),\end{cases}
$$

where $r$ is the intermolecular distance, $a$ is a molecular diameter, $\phi_{a}$ and $\gamma$ are constants. Here, $\gamma$ is set to six to follow the attractive tail of the 12-6 Lennard-Jones intermolecular potential. In terms of a one-particle velocity distribution function, the Enskog-Vlasov equation is expressed as

$$
\frac{\partial f}{\partial t}+\xi_{i} \frac{\partial f}{\partial x_{i}}+\frac{F_{i}\left(x_{i}, t\right)}{m} \frac{\partial f}{\partial \xi_{i}}=C_{E}
$$


The right-hand side of Eq. (14) is the Enskog collision term for dense hard-sphere molecules expressed as

$$
\begin{aligned}
C_{E}=\quad a^{2} \int\left\{Y \left[n \left(x_{i}+\right.\right.\right. & \left.\left.\frac{a}{2} K_{i}, t\right)\right] f\left(x_{i}+a K_{i}, \xi_{1 i}^{\prime}, t\right) f\left(x_{i}, \xi_{i}^{\prime}, t\right)-Y\left[n\left(x_{i}-\frac{a}{2} K_{i}, t\right)\right] \\
& \left.\times f\left(x_{i}-a K_{i}, \xi_{1 i}, t\right) f\left(x_{i}, \xi_{i}, t\right)\right\} H\left(\xi_{r i} K_{i}\right)\left(\xi_{r i} K_{i}\right) \mathrm{d} \boldsymbol{\xi}_{1} \mathrm{~d}^{2} \boldsymbol{K},
\end{aligned}
$$

where $t$ is time, $x_{i}$ is the position $(x, y$, and $z), Y$ is a pair correlation function, $n$ is number density, $K_{i}$ is the unit vector, and $H$ is the Heaviside function. Furthermore, $\xi_{i}$ and $\xi_{1 i}$ indicate the molecular velocity of two colliding molecules, prime ( $I$ ) superscripts indicate quantities of post-collisional molecules, $\xi_{\mathrm{r} i}$ indicates the relative velocity $\xi_{\mathrm{r} i}=\xi_{1 i}-\xi_{i}$, and $F_{i}$ is a self-consistent force field determined from Eq. (13)[28]

$$
F_{i}\left(x_{i}, t\right)=\int_{\left\|x_{1 i}-x_{i}\right\|>a} \frac{\mathrm{d} \phi}{\mathrm{d} r} \frac{x_{1 i}-x_{i}}{\left\|x_{1 i}-x_{i}\right\|} n\left(x_{1 i}, t\right) \mathrm{d} x_{1 i}
$$

where $x_{1 i}$ indicates the general position in the physical space. Once the velocity distribution function $f$ over the whole physical space is obtained as the solution of the Enskog-Vlasov equation, the macroscopic variables, such as density, velocity, and temperature, are respectively defined by

$$
\rho=\int_{-\infty}^{\infty} f \mathrm{~d} \boldsymbol{\xi}, \quad v_{i}=\frac{1}{\rho} \int_{-\infty}^{\infty} \xi_{i} f \mathrm{~d} \boldsymbol{\xi}, \quad T=\frac{1}{3 \rho R} \int_{-\infty}^{\infty}\left(\xi_{i}-v_{i}\right)^{2} f \mathrm{~d} \boldsymbol{\xi}
$$

where $\rho$ is density, $v_{i}=\left(v_{x}, v_{y}, v_{z}\right)$ is velocity, $T$ is temperature, and $\int_{-\infty}^{\infty} \mathrm{d} \boldsymbol{\xi}=\int_{-\infty}^{\infty} \int_{-\infty}^{\infty} \int_{-\infty}^{\infty} \mathrm{d} \xi_{x} \mathrm{~d} \xi_{y} \mathrm{~d} \xi_{z}$.

We utilize the following equation of state[28]:

$$
p=\rho R T \frac{1+\eta+\eta^{2}-\eta^{3}}{(1-\eta)^{3}}-\frac{2}{3} \pi a^{3} \frac{\gamma}{\gamma-3} \frac{\phi_{a}}{k}
$$

where $p$ is pressure, $k$ is the Boltzmann constant, and $\eta=(\pi / 6) n a^{3}$. The first term on the right-hand side of Eq. (17) is obtained from Carnahan and Starling approximation[35] for the equation state of the hard-sphere molecules, and the second term on the right-hand side of Eq. (17) is related to the attractive tail of the Sutherland potential. According to Eq. (17), the critical temperature, $T_{\mathrm{c}}$, is given as follows[28]:

$$
T_{\mathrm{c}}=0.094329 \frac{4 \gamma}{\gamma-3} \frac{\phi_{a}}{k}
$$

The direct simulation Monte Carlo (DSMC) based numerical scheme is used for solving the Enskog-Vlasov equation (EV-DSMC simulation[28, 36]). The DSMC method is a particle scheme for solving the kinetic equation[37, 38]. The EV-DSMC simulation is similar to molecular dynamics as it is a molecular simulation but is essentially different because it is a stochastic simulation. In general, an unsteady molecular simulation requires an ensemble average to estimate the macroscopic variables, such as density, velocity, and temperature; 
hence, the computational cost of the unsteady molecular simulation is exceedingly higher than a steady one. The fluctuation for estimating such a macroscopic variable is to be $O\left(1 /(N M)^{\frac{1}{2}}\right)$, where $N$ is the number of molecules and $M$ is the number of sampling. Barbante et al. [15] showed that the EV-DSMC simulation runs approximately 50-60 times faster than molecular dynamics simulations using the same number of particles, in other words, the EV-DSMC simulation enables us to deal with a larger number of particles than the molecular dynamics simulations in the same computational cost. Hence, we can reduce the fluctuation and obtain the accurate macroscopic variables by utilizing the EV-DSMC simulation for the unsteady net evaporation and condensation problem. Several studies have confirmed that the macroscopic variables obtained from the EV-DSMC simulation show similar tendencies to those obtained from the molecular dynamics simulation for monatomic molecules[15, 24, 25, 26, 27, 28, 29].

\subsection{System setting}

We consider a one-dimensional physical space (z-direction) and three-dimensional molecular velocity space. The system is composed of hard-sphere vapor and its condensed phase (liquid). Figure 2 shows a schematic of the simulation configuration and the spatial distribution of the macroscopic variables at the initial condition $\left(t^{*}=t\left(2 R T_{\mathrm{c}}\right)^{\frac{1}{2}} / a=0\right)$ and the final condition $\left(t^{*}=500\right)$ of the simulation period; the system length is $80 a$ and the total molecular number in the whole system is set to 1.2 million. A region between the two liquid slabs at temperatures $T_{\mathrm{L} 1}$ and $T_{\mathrm{L} 2}$ is filled with vapor, where we refer to the left liquid at $T_{\mathrm{L} 1}$ and right liquid at $T_{\mathrm{L} 2}$ as liquid 1 and liquid 2, respectively. The cell size, $\Delta z / a$, and the time-step size, $\Delta t /\left(a /\left(2 R T_{\mathrm{c}}\right)^{\frac{1}{2}}\right)$, are set to 0.2 and 0.001 , respectively.

In the initial condition $\left(t^{*}=0\right)$, we set the temperatures of liquid 1 and liquid 2 to $0.72 T_{\mathrm{c}}$ and $0.60 T_{\mathrm{c}}$, respectively. As a consequence of the temperature difference between $T_{\mathrm{L} 1}$ and $T_{\mathrm{L} 2}$, net evaporation occurs at the left kinetic boundary and net condensation occurs at the right kinetic boundary; we confirmed that this liquid temperature difference induces relatively strong net evaporation and condensation in our recent study [24].

Figure 2(a) shows the density, velocity in the direction normal to the kinetic boundary, and temperature fields at $t^{*}=0$ estimated from Eq. (16); the higher density regions in the vicinity of $z=-20 a$ and $20 a$ are liquids and the lower density region around the center of the system is vapor. The smooth density transition layers are formed between each liquid and vapor. As can be seen, the vapor velocity in the direction normal to the kinetic boundary (z-direction) takes a positive value, which is induced by net evaporation and condensation. Furthermore, a drop in temperature near the left kinetic boundary occurs as a consequence of net evaporation, while a rise in temperature near the right kinetic boundary occurs as a consequence of net condensation. In Fig. 2(a), we can observe a peak in temperature in the vicinity of the kinetic boundary with net condensation. This peak in temperature is also observed in the previous studies $[24,27,39]$ by both molecular dynamics and EVDSMC simulations. A detailed physical explanation of this peak in temperature has not been investigated; however, it is confirmed that this peak in temperature occurs only when the temperature difference between the kinetic boundary and liquid is larger, that is, the case of strong net condensation. 
Figure 2(b) shows the density, velocity in the direction normal to the kinetic boundary, and temperature fields at $t^{*}=500$ estimated from Eq. (16). As can be seen, a velocity in the direction normal to the kinetic boundary ( $z$-direction) becomes zero in the whole system. The temperature field in the whole system becomes uniform and constant as a result of the drop and rise in temperature attributed to the net evaporation and condensation. These results indicate that the vapor-liquid two-phase system achieves at vapor-liquid equilibrium at $t^{*}=500$.

In addition, we estimate the spatial distribution of the macroscopic variables in Fig. 2 from the ensemble average of 200 simulations obtained from different initial conditions. To obtain each initial condition with a different microstate, we simulate steady net evaporation and condensation between two liquid slabs at different temperatures using velocity-scaling and particle-shifting methods[24, 27]; The velocity-scaling method modifies the molecular velocity in the bulk liquid at each time step, keeping the bulk liquid temperatures at $0.72 T_{\mathrm{c}}$ and $0.60 T_{\mathrm{c}}$. The particle-shifting method modifies the position of molecules in the whole system, fixing the position of the kinetic boundary during the simulation period.

Figure 3 shows the temporal evolution of the net mass flux $\rho v_{z}$ at each kinetic boundary, where $\rho v_{z}$ is obtained as the difference between $J_{\text {out }}$ and $J_{\text {coll }}$. The molecular mass flux relations (Eq. (5)) and the definitions of $\rho v_{z}$ in the simulation system of this study are illustrated in the enlarged view of Fig. 2. We define the position of each kinetic boundary to be $2.5 \delta$ from the center of each 10-90 thickness density transition layer toward the vapor, at which the vapor density becomes the saturated value at liquid temperature in vapor-liquid equilibrium, where $\delta$ is the thickness of the density transition layer. As can be seen, $\rho v_{z}$ at each kinetic boundary decreases drastically until $t^{*}=200$. After that time $\rho v_{z}$ at each kinetic boundary decreases gently and converges to zero eventually.

\section{Results and discussion}

\subsection{Confirmation of the normalized velocity distribution function.}

In this section, we confirm whether the functional form of the KBC in unsteady net evaporation/condensation at each time is the same as that in steady net evaporation/condensation. In other words, we confirm the normalized velocity distribution function of the KBC $(\hat{f}$ in Eq. (11) or (12)) to be the normalized Maxwellian distribution at liquid temperature expressed in Eq. (2) during unsteady net evaporation/condensation. We postulate the position of the boundary between the bulk liquid and interface layer to specify the thickness of the interface layer (see Fig. 2). Herewith, we can estimate a delay time caused by molecules traveling in the interface layer.

Figure 4 shows the temperature field at the initial condition $\left(t^{*}=0\right)$ : the solid line denotes temperature estimated from Eq. (16), the dotted line denotes temperature in the direction tangential to the kinetic boundary ( $x$-direction), estimated as $T_{x}=\frac{1}{\rho R} \int_{-\infty}^{\infty} \xi_{x}^{2} f \mathrm{~d} \boldsymbol{\xi}$, and the dashed line denotes temperature in the direction normal to the kinetic boundary (z-direction), estimated as $T_{z}=\frac{1}{\rho R} \int_{-\infty}^{\infty}\left(\xi_{z}-v_{z}\right)^{2} f \mathrm{~d} \boldsymbol{\xi}$. We emphasize that the tendencies of these temperatures $\left(T, T_{x}\right.$, and $\left.T_{z}\right)$ are in good agreement with these obtained from the 
molecular dynamics simulation[40, 41]. These are important results showing the validity of the EV-DSMC simulation.

In Fig. 4, $B_{\mathrm{L} 1}$ and $B_{\mathrm{L} 2}$ indicate the positions of the boundaries between each bulk liquid and each interface layer. These positions are set to 3.0a from each center of the 10-90 thickness density transition layer toward each liquid. As can be seen, $T_{x}$ and $T_{z}$ agree with $T$, showing that temperature in each bulk liquid is isotropic. In addition, $B_{\mathrm{V} 1}$ and $B_{\mathrm{V} 2}$ are the positions of each kinetic boundary as defined in Section 2.3. The distance between $B_{\mathrm{L} 1}$ and $B_{\mathrm{V} 1}$ or $B_{\mathrm{L} 2}$ and $B_{\mathrm{V} 2}$ represents the thickness of the interface layer as illustrated in Fig. 1. The delay time, $t_{\mathrm{I}}$, caused by molecules traveling in the interface layer can be expressed from the characteristic velocity, $\left(2 R T_{0}\right)^{\frac{1}{2}}$, at the reference temperature $T_{0}=\left(T_{\mathrm{L} 1}+T_{\mathrm{L} 2}\right) / 2=0.66 T_{\mathrm{c}}$ and the thickness of the interface layer, $L_{\mathrm{I}}$, as $t_{\mathrm{I}}=L_{\mathrm{I}} /\left(2 R T_{0}\right)^{\frac{1}{2}}$. Since $L_{\mathrm{I}}$ of each interface layer is approximately $6 a$ or $8 a$ at $t^{*}=0$ found from Fig. 4 , the delay time normalized by the molecular diameter and the critical temperature, $t_{\mathrm{I}}^{*}=t_{\mathrm{I}}\left(2 R T_{\mathrm{c}}\right)^{\frac{1}{2}} / a$, becomes approximately 7.4 or 9.8 , that is, $t_{\mathrm{I}}^{*} \approx 10$.

Then, we examine the normalized velocity distribution functions at $B_{\mathrm{V} 1}, B_{\mathrm{V} 2}, B_{\mathrm{L} 1}$, and $B_{\mathrm{L} 2}$. In the EV-DSMC simulation, the normalized velocity distribution function is obtained by the following procedure. We set the upper and lower limits of the velocity space to $\xi_{i} /\left(2 R T_{\text {ref }}\right)^{\frac{1}{2}}=-6$ and $\xi_{i} /\left(2 R T_{\text {ref }}\right)^{\frac{1}{2}}=6$, respectively, where $T_{\text {ref }}$ is set to $0.60 T_{\mathrm{c}}$, and divide this velocity space into 60 cells. We can obtain the normalized velocity distribution function at the arbitrary cell of the physical space in the simulation system, which is divided into 400 cells, by determining which cells molecular velocity belongs to and counting the number of molecules[21]. As with the estimation of the macroscopic variables, the normalized velocity distribution function is estimated from the ensemble average of 200 simulations obtained from different initial conditions.

Figure 5 shows the normalized velocity distribution function $\left(F=\left(2 R T_{\text {ref }}\right)^{\frac{1}{2}} \hat{f}\right)$ composed of molecules outgoing from $B_{\mathrm{L} 1}$ or $B_{\mathrm{V} 1}$ and having the positive velocity in the $z$-direction at $t^{*}=0,100,200$ and 500. The closed circles denote $F$ composed of molecules outgoing from $B_{\mathrm{L} 1}$ into the interface layer, and the open circles denote $F$ composed of molecules outgoing from $B_{\mathrm{V} 1}$ into the vapor phase, that is, the normalized velocity distribution functions of the KBCs because $B_{\mathrm{V} 1}$ indicates the position of the kinetic boundary of liquid 1 . In Fig. 5, the dashed lines denote the normalized Maxwellian distribution at $T_{\mathrm{L} 1}$, obtained as

$$
F^{*}\left(T_{\mathrm{L} 1}\right)=\left(\frac{T_{\mathrm{ref}}}{\pi T_{\mathrm{L} 1}}\right)^{\frac{1}{2}} \exp \left(-\frac{\xi_{i}^{2}}{2 R T_{\mathrm{ref}}} \frac{T_{\mathrm{ref}}}{T_{\mathrm{L} 1}}\right),
$$

where $\xi_{i}$ indicates the molecular velocity in the direction tangential $(i=x)$ or normal $(i=z)$ to the kinetic boundary.

As can be seen in Fig. 5, the closed circles are in excellent agreement with the dashed lines at each normalized time. This result shows that the normalized velocity distribution function outgoing from $B_{\mathrm{L} 1}$ into the interface layer are well described by the normalized Maxwellian distribution at $T_{\mathrm{L} 1}$. In contrast, the open circles slightly differ from the dashed lines at $t^{*}=0$. This result shows that the number of molecules that have a molecular velocity near $\xi_{x} /\left(2 R T_{\text {ref }}\right)^{\frac{1}{2}}=0$ is larger than the normalized Maxwellian distribution at $T_{\mathrm{L} 1}$ and 
the normalized velocity distribution function outgoing from $B_{\mathrm{V} 1}$ becomes an anisotropic velocity distribution function. We confirm that the second moment of the normalized velocity distribution function outgoing from $B_{\mathrm{V} 1}$ in the $x$-direction $T_{\mathrm{t}}$,

$$
T_{\mathrm{t}}=\frac{1}{\rho_{\text {out }} R} \int_{\xi_{z}>0} \xi_{x}^{2} f_{\text {out }} \mathrm{d} \boldsymbol{\xi}
$$

where $\rho_{\text {out }}=\int_{\xi_{z}>0} f_{\text {out }} \mathrm{d} \boldsymbol{\xi}$, is $0.67 T_{\mathrm{c}}$, which is smaller than the temperature of liquid 1 $0.72 T_{\mathrm{c}}$. This tendency has also been observed in a vapor-liquid two-phase system with strong net evaporation by the numerical simulation based on molecular dynamics[39]. In this simulation, the open circles eventually agree with the dashed lines because net evaporation becomes weak with time.

Figure 6 shows the normalized velocity distribution function $\left(F=\left(2 R T_{\text {ref }}\right)^{\frac{1}{2}} \hat{f}\right)$ composed of molecules outgoing from $B_{\mathrm{L} 2}$ or $B_{\mathrm{V} 2}$ and having the negative velocity in the $z$-direction at $t^{*}=0,100,200$ and 500. The closed circles denote $F$ composed of molecules outgoing from $B_{\mathrm{L} 2}$ into the interface layer, and the open circles denote $F$ composed of molecules outgoing from $B_{\mathrm{V} 2}$ into the vapor phase, that is, the normalized velocity distribution functions of the KBCs because $B_{\mathrm{V} 2}$ indicates the position of the kinetic boundary of liquid 2 . In Fig. 6, the dashed lines denotes the normalized Maxwellian distribution at $T_{\mathrm{L} 2}$, obtained as

$$
F^{*}\left(T_{\mathrm{L} 2}\right)=\left(\frac{T_{\mathrm{ref}}}{\pi T_{\mathrm{L} 2}}\right)^{\frac{1}{2}} \exp \left(-\frac{\xi_{i}^{2}}{2 R T_{\mathrm{ref}}} \frac{T_{\mathrm{ref}}}{T_{\mathrm{L} 2}}\right),
$$

where $\xi_{i}$ indicates the molecular velocity in the direction tangential $(i=x)$ or normal $(i=z)$ to the kinetic boundary.

As can be seen in Fig. 6, similar to Fig. 5, the closed circles are in excellent agreement with the dashed lines at each normalized time. This result shows that the normalized velocity distribution function outgoing from $B_{\mathrm{L} 2}$ into the interface layer are well described by the normalized Maxwellian distribution at $T_{\mathrm{L} 2}$. In contrast, the open circles slightly differ from the dashed lines at $t^{*}=0$. Contrary to Fig. 5 , this result shows that the number of molecules that have a molecular velocity near $\xi_{x} /\left(2 R T_{\text {ref }}\right)^{\frac{1}{2}}=0$ is smaller than the normalized Maxwellian distribution at $T_{\mathrm{L} 2}$ and the normalized velocity distribution function outgoing from $B_{\mathrm{V} 2}$ becomes an anisotropic velocity distribution function. We confirm that the second moment of the normalized velocity distribution function outgoing from $B_{\mathrm{V} 2}$ in the $x$-direction $T_{\mathrm{t}}$,

$$
T_{\mathrm{t}}=\frac{1}{\rho_{\text {out }} R} \int_{\xi_{z}<0} \xi_{x}^{2} f_{\text {out }} \mathrm{d} \boldsymbol{\xi}
$$

where $\rho_{\text {out }}=\int_{\xi_{z}<0} f_{\text {out }} \mathrm{d} \boldsymbol{\xi}$, is $0.65 T_{\mathrm{c}}$, which is larger than the temperature of liquid $20.60 T_{\mathrm{c}}$. This tendency has also been observed in vapor-liquid two-phase systems with strong net condensation by the numerical simulation based on molecular dynamics and the EV-DSMC simulation $[22,24,39]$. Similar to Fig. 5, the open circles eventually agree with the dashed lines because net condensation becomes weak with time. 
On the basis of Figs. 5 and 6, the normalized velocity distribution functions outgoing from $B_{\mathrm{L} 1}$ and $B_{\mathrm{L} 2}$ into the interface layer are well described as the normalized Maxwellian distributions at $T_{\mathrm{L} 1}$ and $T_{\mathrm{L} 2}$ defined in Eq. (2), while the normalized velocity distribution functions outgoing from $B_{\mathrm{V} 1}$ and $B_{\mathrm{V} 2}$ into the vapor phase become an anisotropic velocity distribution functions at $t^{*}=0$. However, the normalized velocity distribution functions outgoing from $B_{\mathrm{V} 1}$ and $B_{\mathrm{V} 2}$ into the vapor phase correspond to the normalized Maxwellian distributions at $T_{\mathrm{L} 1}$ and $T_{\mathrm{L} 2}$ with time. We conclude that the normalized velocity distribution functions of the KBCs (Eqs. (11) and (12)) can be assumed to be the normalized Maxwellian distribution in unsteady net evaporation and condensation even though the interface layer between the bulk liquid and kinetic boundary has a finite thickness and the delay time, $t_{\mathrm{I}}^{*} \approx 10$, arises from molecules traveling in this finite interface layer.

\subsection{Estimation of liquid temperature}

As stated in Section 2.1, we can impose the KBC (Eq. (11) or (12)) by simply specifying liquid temperature in steady net evaporation/condensation. In this section, we estimate the temporal evolution of liquid temperatures $T_{\mathrm{L} 1}$ and $T_{\mathrm{L} 2}$ to establish the $\mathrm{KBC}$, which depends on liquid temperature, in unsteady net evaporation/condensation.

We examine the process of estimating liquid temperature. The enlarged view of Fig. 4 shows the temperature field in liquid 2 from $t^{*}=0$ to 200; we focus only on liquid 2 because its temperature change is larger than that of liquid 1 (see Fig. 7: a detailed explanation is given in the next paragraph). In general, liquid temperature changes spatiotemporally with time on the basis of a one-dimensional heat conduction equation; hence, we should estimate the liquid temperature at position $B_{\mathrm{L} 1}$ or $B_{\mathrm{L} 2}$ to specify the KBC. In contrast, the bulk liquid temperature of liquid 2 changes, keeping a spatially almost uniform distribution with time, as shown in the enlarged view of Fig. 4.

To examine the accuracy of this spatially uniform temperature distribution, we analytically solve the one-dimensional heat conduction equation with the temporal evolution of temperature of liquid 2 fitted by the exponential function, and then we define the index of uniformity of the spatial temperature distribution. As a result, we confirm that the spatial temperature distribution is to be approximately spatially uniform because the characteristic time of thermal diffusion in liquid $2\left(t_{\mathrm{d}}^{*}=t_{\mathrm{d}}\left(2 R T_{\mathrm{c}}\right)^{\frac{1}{2}} / a=2.00\right.$ in this study) is much smaller; therefore, we can estimate each liquid temperature as the spatial average. A detailed explanation of thermal diffusion in liquid 2 is given in Appendix A.

Figure 7 shows the temporal evolution of each liquid temperature. Similar to the temporal evolution of the net mass flux $\rho v_{z}$ shown in Fig. 3, each liquid temperature changes drastically until $t^{*}=200$; after that time, each liquid temperature changes gently and eventually converges to approximately $0.69 T_{\mathrm{c}}$. It is also found that the temperature change of liquid 2 is larger than that of liquid 1 . In this simulation, we observe the maximum local Mach number $(|\mathrm{Ma}| \approx 0.2)$ at $B_{\mathrm{V} 2}$ of the initial condition $\left(t^{*}=0\right)$. We expect that this maximum Mach number causes the larger temperature change in liquid 2. 


\subsection{Validation of the $K B C$ during unsteady net evaporation/condensation}

In this section, we carry out a numerical simulation of the Boltzmann equation with the $\mathrm{KBC}$ that varies with the temporal evolution of liquid temperature and then validate the accuracy of the KBC by comparing the macroscopic variables, velocity and temperature, obtained from the numerical simulation of the Boltzmann equation and those obtained from the EV-DSMC simulation at each time. Because it has been found that the KBC significantly affects the macroscopic variables in vapor between two liquid slabs at different temperatures $[40,41,42]$, a proper specification of the KBC is critical. In other words, the KBC in net evaporation/condensation is valid if and only if the macroscopic variables in vapor obtained from these two simulations agree with high accuracy at each time. This simple and practical validation method has often been performed in the numerical simulation based on molecular dynamics[40, 41].

In the simulation of the Boltzmann equation, we consider a one-dimensional physical space (z-direction) and three-dimensional molecular velocity space. The system is composed of the hard-sphere vapor between two kinetic boundaries at temperatures $T_{\mathrm{L} 1}$ and $T_{\mathrm{L} 2}$. We utilize the ES-BGK model Boltzmann equation (ES-BGK equation)[43], which can set the Prandtl number of hard-sphere molecules as 0.66[30], expressed as

$$
\frac{\partial f}{\partial t}+\xi_{i} \frac{\partial f}{\partial x_{i}}=\frac{p}{\mu(1-\nu)}[G(f)-f]
$$

where $\mu$ is the viscosity coefficient and $\nu$ is a constant for fitting the Prandtl number,

$$
\operatorname{Pr}=\frac{1}{1-\nu},
$$

and set to $1 / 2$ in this simulation. In the right-hand side of Eq. (23), $G(f)$ is given as

$$
G(f)=\frac{\rho}{\left(\operatorname{det}\left(2 \pi \Upsilon_{i j}\right)\right)^{\frac{1}{2}}},
$$

where $\Upsilon_{i j}$ is

$$
\Upsilon_{i j}=(1-\nu) R T \delta_{i j}+\nu \Phi_{i j}
$$

where $\delta_{i j}$ is Kronecker's delta and $\rho \Phi_{i j}$ is the stress tensor.

The system length, $L_{\mathrm{V}}$, of this simulation is determined as $L_{\mathrm{V}}=\ell_{0} / \mathrm{Kn}$, where $\ell_{0}$ is the mean free path at the mean temperature of two liquid slabs at $t^{*}=0: T_{0}=0.66 T_{\mathrm{c}}$. The mean free path $\ell_{0}$ and the Knudsen number are estimated by using the result of the EV-DSMC simulation at $t^{*}=0$. The system length is approximately constant with time in the EVDSMC simulation because the position of the kinetic boundary with net evaporation goes away from another kinetic boundary with net condensation, while that with net condensation goes toward another kinetic boundary with net evaporation; therefore, we assume $L_{\mathrm{V}}$ to be constant in the numerical simulation of the ES-BGK equation.

The finite difference method is used for the numerical scheme. The cell numbers of the physical space and the velocity space are set to 500 and 2000, respectively; the cell size 
of the physical space, $\Delta z / \ell_{0}$, is set to $1.0 \times 10^{-4}$ and the cell size of the velocity space, $\Delta \xi_{z} /\left(2 R T_{0}\right)^{\frac{1}{2}}$, is set to $1.0 \times 10^{-4}$ near $\xi_{z}=0$ and $1.0 \times 10^{-2}$ far from $\xi_{z}=0$. Note that we performed this simulation using double cell numbers of physical space and the velocity space and confirmed that the result of the macroscopic variables shows no dependency on the cell numbers. More detailed explanations of the ES-BGK equation and numerical scheme can be found in the literature[24, 43, 44].

In the simulation of the ES-BGK equation, we impose Eq. (11) as the KBC at the kinetic boundary with net evaporation ( $B_{\mathrm{V} 1}$ in Fig. 4), and Eq. (12) as the KBC at the kinetic boundary with net condensation ( $B_{\mathrm{V} 2}$ in Fig. 4). The values of $\beta_{\mathrm{ne}}$ and $\beta_{\mathrm{nc}}$ in Eqs. (11) and (12) vary with the temporal evolution of liquid temperature as shown in Fig. 7 ; the liquid temperature dependence of $\beta_{\mathrm{ne}}$ and $\beta_{\mathrm{nc}}$ are explicitly clarified[27], and these dependence fitted by the linear regression analysis are given as follows:

$$
\beta_{\mathrm{ne}}\left(T_{\mathrm{L}}\right)=-0.7411\left(T_{\mathrm{L}} / T_{\mathrm{c}}\right)+1.315, \quad \beta_{\mathrm{nc}}\left(T_{\mathrm{L}}\right)=-1.024\left(T_{\mathrm{L}} / T_{\mathrm{c}}\right)+1.514 .
$$

We utilize Eq. (27) to specify the values of $\beta_{\text {ne }}$ and $\beta_{\text {nc }}$ at each time.

Figure 8 shows the results of the comparison between the vapor velocity and temperature fields obtained from the numerical simulation of the ES-BGK equation and the EV-DSMC simulation. The open circles denote the results of the EV-DSMC simulation and the solid lines denote those of the numerical simulation of the ES-BGK equation. Note that we added the proper offset to the data for legibility. As shown in Fig. 8 (a), the velocity fields in vapor obtained from these two simulations are in excellent agreement at each time. In contrast, as shown in Fig. 8 (b), the slope of the temperature field at $t^{*}=0$ obtained from the EV-DSMC simulation is slightly larger than that obtained from the numerical simulation of the ES-BGK equation; the temperature at $B_{\mathrm{V} 1}$ obtained from the EV-DSMC simulation is smaller, and that at $B_{\mathrm{V} 2}$ is larger.

As was mentioned in Section 3.1, $T_{\mathrm{t}}$, that is, the second moment of the normalized velocity distribution function in the $x$-direction, at $B_{\mathrm{V} 1}$ estimated from Eq. (20) is smaller than the temperature of liquid $10.72 T_{\mathrm{c}}$ at $t^{*}=0$, while that at $B_{\mathrm{V} 2}$ estimated from Eq. (22) is larger than the temperature of liquid $20.60 T_{\mathrm{c}}$ at $t^{*}=0$. These results imply that the difference of the temperature fields between two different simulations is related to the anisotropic velocity distribution function of the KBC (see Figs. 5 and 6). In our recent study[24], we also confirmed that the anisotropic velocity distribution function of the KBC affects the temperature gradient in vapor in the vapor-liquid two-phase system with strong net evaporation/condensation. The difference of the temperature field at $t^{*}=0$, however, is sufficiently small and decreases with time as shown in Fig. 8 (a).

On the basis of these results, we conclude that the macroscopic variables in the vapor phase obtained from the numerical simulation of the ES-BGK equation and EV-DSMC simulation are in good agreement. Therefore, the KBC (Eq. (11) or (12)) that depends on liquid temperature is guaranteed to be accurate even in unsteady net evaporation and condensation by considering the time evolution of liquid temperature.

Here, we briefly summarize a comparison of the numerical simulation of the ES-BGK equation and the EV-DSMC simulation. Note that since the EV-DSMC simulation is categorized into the particle scheme, which does not directly solve the Enskog-Vlasov equation, 
as explained in Section 2.3, it is difficult to make a comparison of these algorithms. However, as far as we examine the computational time in the present simulation settings, the numerical simulation of the ES-BGK equation with the KBC runs approximately 40 times faster than the EV-DSMC simulation to obtain one simulation. As was mentioned in Section 2.3, the macroscopic variables, such as vapor velocity and temperature shown in Fig. 8, are estimated from the ensemble average of one simulation obtained from different initial conditions in the EV-DSMC simulation; thus, the computational cost of the numerical simulation of the ES-BGK equation is obviously smaller than the EV-DSMC simulation. In regard to this point, the numerical simulation of the Boltzmann equation or its model equation with a certain $\mathrm{KBC}$, which is established or validated by the molecular simulation, is useful to examine the vapor-liquid two-phase flow with net evaporation/condensation.

\section{Conclusion}

In this study, we examined the $\mathrm{KBC}$, which depends on liquid temperature, in the vapor-liquid two-phase system with unsteady net evaporation and condensation. First, we simulated an unsteady net evaporation and condensation problem in the system composed two liquid slabs at different temperatures by using the molecular simulation based on meanfield kinetic theory, and then we confirmed whether the functional form of the KBC at each time is the same as that in steady net evaporation/condensation. The results showed that the KBC follows the temporal evolution of liquid temperature attributed to unsteady net evaporation and condensation even though the interface layer between the bulk liquid and kinetic boundary has a finite thickness and the delay time arises from molecules traveling in this finite interface layer. Finally, we carried out a numerical simulation of the Boltzmann equation, and then validated the accuracy of the KBC by comparing the macroscopic variables, such as vapor velocity and temperature, obtained from the numerical simulation of the Boltzmann equation with those of the molecular simulation based on mean-field kinetic theory. The results showed that the KBC is applicable to a vapor-liquid two-phase system with unsteady net evaporation and condensation.

We now comment on the applicable limits of the results of this study. To apply the results of this study, we should consider the relatively gentle temperature change of the bulk liquid; specifically, the maximum local Mach number $(|\mathrm{Ma}| \approx 0.2)$ in this study was observed at $B_{\mathrm{V} 2}$ of the initial condition $\left(t^{*}=0\right)$. If the maximum local Mach number is larger, the temporal variation of the macroscopic variable, such as liquid temperature, becomes drastic. We expect that the normalized velocity distribution function of the KBC does not follow the temporal evolution of liquid temperature when the characteristic time of the liquid temperature variation, becomes larger than the delay time ( $t_{\mathrm{I}}^{*} \approx 10$ in this study) caused by molecules traveling in the interface. However, the maximum Mach number of the vapor velocity induced by net evaporation is limited to $|\mathrm{Ma}| \approx 0.6$, which has been observed in the case of evaporation into vacuum[21]. Furthermore, the maximum Mach number of the vapor velocity induced by net condensation is also limited to $|\mathrm{Ma}| \approx 0.6$, which is caused by the non-unity evaporation/condensation coefficients and called the blocking effect[45]. In fact, the evaporation into a vacuum and blocking effect are extreme situations of net 
evaporation and condensation; hence, we conclude that the results proposed in this study can be applied to the practical problem of vapor-liquid two-phase system with unsteady net evaporation/condensation. 


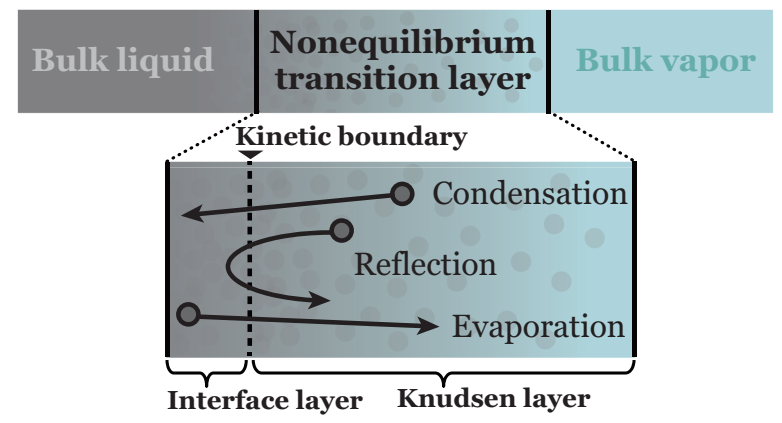

Figure 1: Picture of the vapor-liquid two-phase system.
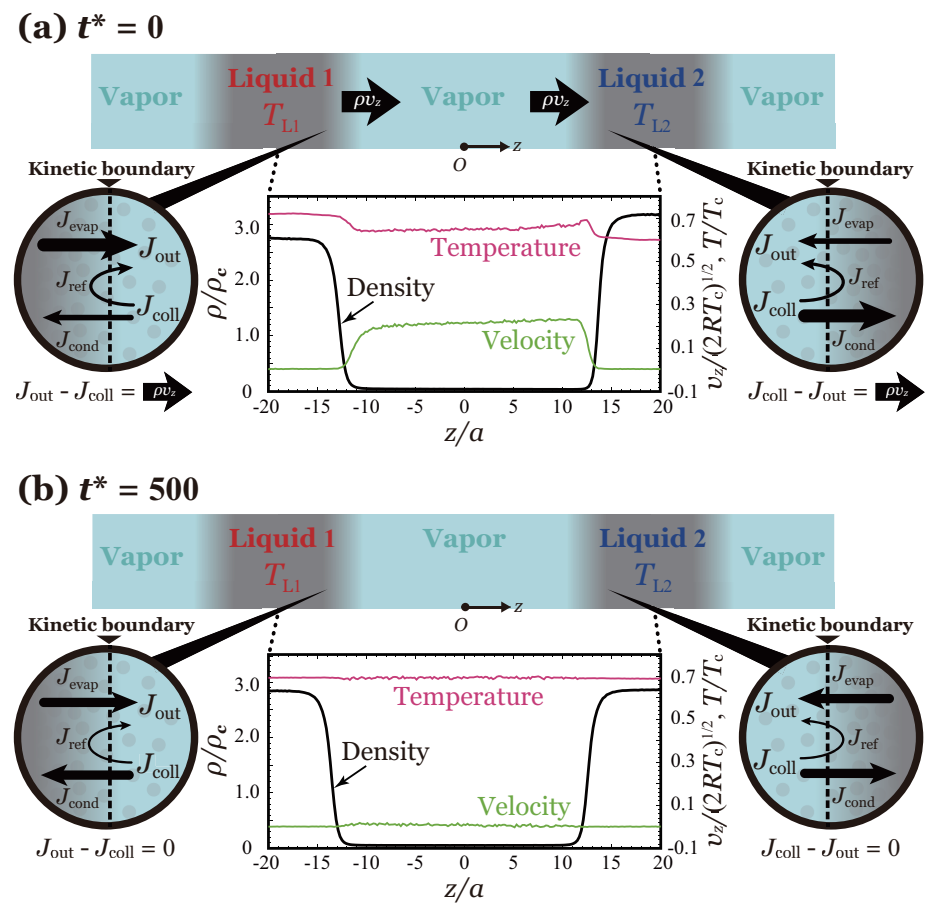

Figure 2: Schematic of the simulation configuration and density, velocity, and temperature fields normalized by each critical value at the (a) initial condition $\left(t^{*}=0\right)$ and $(\mathrm{b})$ final condition $\left(t^{*}=500\right)$. 


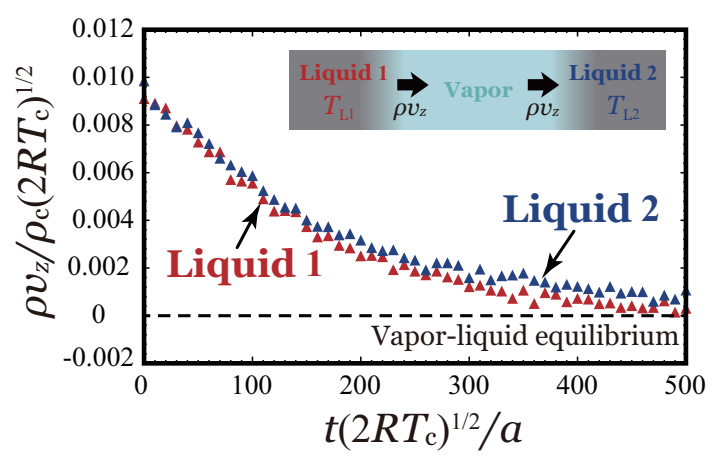

Figure 3: Temporal evolution of the net mass flux $\rho v_{z}$ at each kinetic boundary.



Figure 4: Various temperature fields at the initial condition $\left(t^{*}=0\right)$ : (enlarged view) the temporal evolution of temperature fields in liquid 2 , where $\chi$ is the spatial coordinate in liquid $2\left(\chi=0\right.$ is the position of $B_{\mathrm{L} 2}$ and $\chi=1$ is the end of liquid 2).
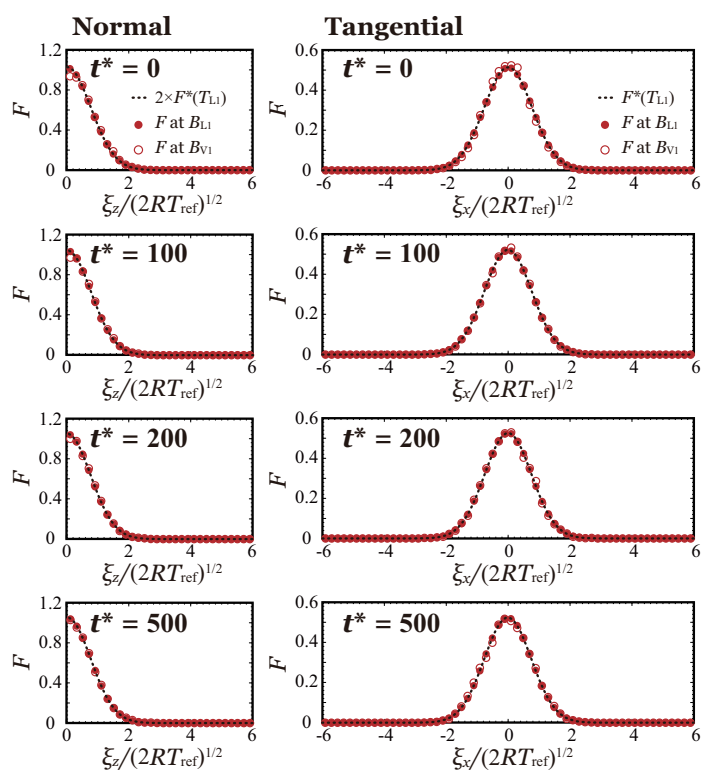

Figure 5: Velocity distribution functions composed of the molecules outgoing from the liquid into vapor phase $\left(\xi_{z}>0\right)$ at $B_{\mathrm{L} 1}$ and $B_{\mathrm{V} 1}$. 

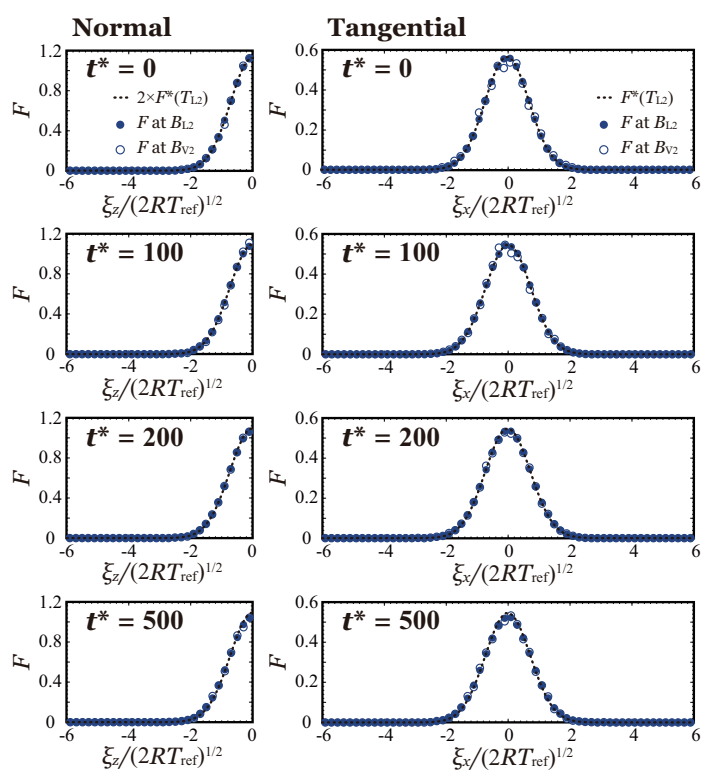

Figure 6: Velocity distribution functions composed of the molecules outgoing from the liquid into vapor phase $\left(\xi_{z}<0\right)$ at $B_{\mathrm{L} 2}$ and $B_{\mathrm{V} 2}$.

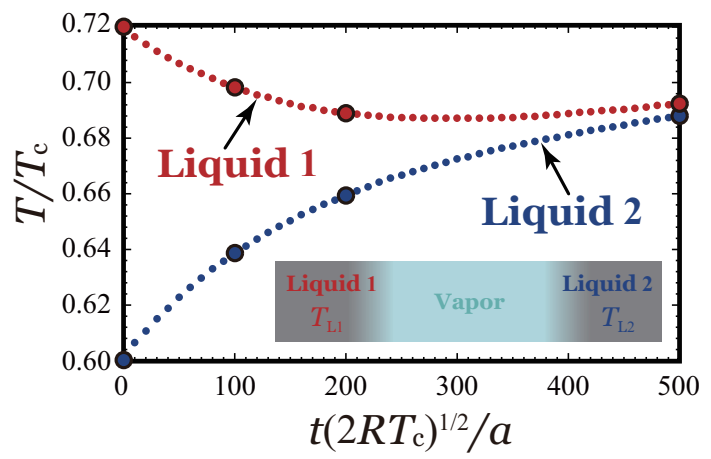

Figure 7: Temporal evolution of the temperatures of liquid 1 and liquid 2. 
(a) Velocity

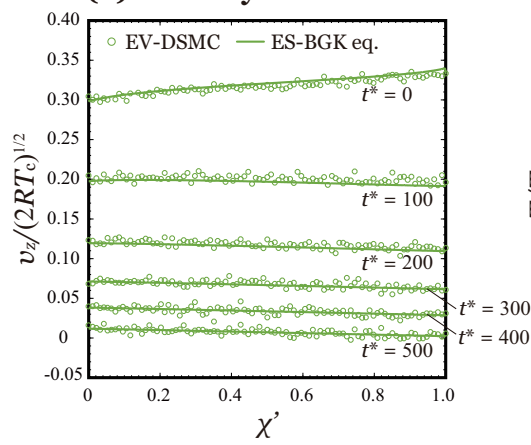

(b) Temperature

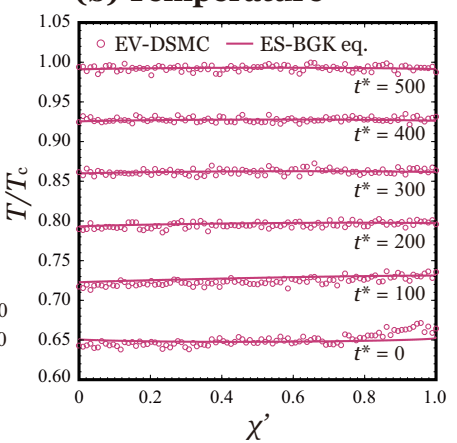

Figure 8: Comparison of the macroscopic variables in the vapor phase obtained from the EV-DSMC simulation and the numerical simulation of the ES-BGK equation: (a) velocity fields at each time and (b) temperature fields at each time, where $\chi^{\prime}$ is the spatial coordinate in vapor $\left(\chi^{\prime}=0\right.$ is the position of $B_{\mathrm{V} 1}$ and $\chi^{\prime}=1$ is that of $B_{\mathrm{V} 2}$ ). 


\section{Appendix A. Thermal diffusion in a medium bounded by two parallel planes}

Our proposed KBC (Eqs. (11) and (12)) requires liquid temperature $T_{\mathrm{L}}$. It is widely accepted that the temperature field in liquid phase can be assumed to be uniform in the case of steady net evaporation/condensation[22, 24, 25, 26, 27, 46]; hence, $T_{\mathrm{L}}$ can be defined without ambiguity. However, the uniformity of the temperature field is no longer necessarily guaranteed in the case of unsteady net evaporation/condensation; hence, we need to evaluate $T_{\mathrm{L}}$ with extra caution. In Section 3.2, we estimate the spatially average temperature $T_{\mathrm{A}}$ over the bulk liquid and then define this average to be the liquid temperature $T_{\mathrm{L}}$. This definition can be accepted only if the temperature field is uniform enough. In this appendix, we discuss the legitimacy of this definition.

Under the assumption of no advection, the unsteady temperature field of the bulk liquid is governed by the one-dimensional heat conduction equation:

$$
\frac{\partial T}{\partial t}=\theta \frac{\partial^{2} T}{\partial z^{2}}
$$

where $\theta$ is thermal diffusivity, which can be obtained with the use of a Chapman-Enskog like successive approximation of the Enskog-Vlasov equation[34],

$$
\theta=\frac{\lambda}{C \rho}=A\left(\frac{R T}{\pi}\right)^{\frac{1}{2}} \frac{m a^{2}}{\rho},
$$

where $C$ is the specific heat with constant pressure and $\lambda$ is heat conductivity. We used the following expression of $A$ in Eq. (A.2):

$$
A=\frac{0.8006}{Y(\eta)}\left[1+4.800(\eta Y(\eta))+12.12(\eta Y(\eta))^{2}\right], \quad \eta=\frac{\pi}{6} \frac{\rho a^{3}}{m},
$$

where $Y(\eta)=(1-\eta) /\left(2(1-\eta)^{3}\right)[28,34,36]$. The liquid thermal diffusivity $\theta=5.77 a\left(2 R T_{\mathrm{c}}\right)^{\frac{1}{2}}$ is obtained at $0.60 T_{\mathrm{c}}$ from Eq. (A.2) The temporal bulk liquid temperature field obtained from Eq. (A.1) with appropriate boundary and initial conditions, is in general spatially nonuniform; however, the bulk liquid temperature of liquid 2 obtained from the EV-DSMC simulation changes while keeping a spatially almost uniform distribution as shown in the enlarged view of Fig. 4. Note that we also observed that the bulk liquid temperature of liquid 1 similarly changes while keeping a spatially almost uniform distribution. These results suggest that the use of $T_{\mathrm{A}}$ for $T_{\mathrm{L}}$ may be accepted in the range of the present calculation. We further discuss the legitimacy the use of $T_{\mathrm{A}}$ for $T_{\mathrm{L}}$ by considering the solution of Eq. (A.1).

To investigate the temporal change of the spatial temperature distribution in the liquid, we consider the following problem, taken from the textbook of Carslaw and Jaegar [47]. Here, the bulk liquid is bounded by two parallel planes: the region $-L<z<L$ with zero initial temperature, and the temperature of surface $T_{\mathrm{S}}$ changes with time. Note that here, the uniformity of the spatial temperature distribution greatly depends on the rate of change of $T_{\mathrm{S}}$. In order to solve Eq. (A.1), we properly prepare the boundary condition $T_{\mathrm{S}}$. We assume that $T_{\mathrm{S}}$ can be roughly approximated by $T_{\mathrm{A}}$. 
The temporal change of $T_{\mathrm{A}}$ is shown in Fig. A.9. We found that the data can be well fitted by the exponential function:

$$
T_{\mathrm{S}}(t: \omega)=T_{0}[1-\exp (-\omega t)]
$$

where $t$ is time, $T_{0}$ is the temperature difference and $\omega$ is the fitting parameter, where $0.005 \leq \omega \leq 0.008$. The temperature distribution with the boundary condition (Eq. (A.4)) can be found on p.104 of the literature[47]:

$$
\begin{aligned}
T(t ; \omega)= & T_{0}-T_{0} \exp (-\omega t) \frac{\cos (x \sqrt{\omega / \theta})}{\cos (L \sqrt{\omega / \theta})} \\
& -\frac{16 T_{0} \omega L^{2}}{\pi} \sum_{n=0}^{\infty} \frac{(-1)^{n} \exp \left[-(2 n+1)^{2} \pi^{2} \theta t /\left(4 L^{2}\right)\right]}{(2 n+1)\left[4 \omega L^{2}-(2 n+1)^{2} \pi^{2} \theta\right]} \cos \left[\frac{(2 n+1) \pi x}{2 L}\right] .
\end{aligned}
$$

We introduce the characteristic time $t_{\mathrm{d}}$ :

$$
t_{\mathrm{d}}=\frac{L^{2}}{\theta}
$$

and the following nondimensional variables:

$$
T^{*}=\frac{T}{T_{0}}, \quad T_{\mathrm{S}}^{*}=\frac{T_{\mathrm{S}}}{T_{0}}, \quad \tau=\frac{t}{t_{\mathrm{d}}}, \quad \zeta=\frac{z}{L}, \quad b=\omega t_{\mathrm{d}} .
$$

Then, Eqs. (A.4) and (A.5) can be rewritten as

$$
\begin{gathered}
T_{\mathrm{S}}^{*}(\tau ; b)=1-\exp (-b \tau), \\
T^{*}(\tau ; b)=1-\exp (-b \tau) \frac{\cos (\zeta \sqrt{b})}{\cos (\sqrt{b})} \\
+\frac{16 b}{\pi} \sum_{n=0}^{\infty} \frac{(-1)^{n} \exp \left[-(2 n+1)^{2} \pi^{2} \tau / 4\right]}{(2 n+1)\left[(2 n+1)^{2} \pi^{2}-4 b\right]} \cos \left[\frac{(2 n+1) \pi \zeta}{2}\right] .
\end{gathered}
$$

Integration of Eq. (A.9) on $0 \leq \zeta \leq 1$ gives the average temperature $T_{\mathrm{A}}^{*}$ :

$$
T_{\mathrm{A}}^{*}(\tau ; b)=1-\exp (-b \tau) \frac{\sin (\sqrt{b})}{\sqrt{b} \cos (\sqrt{b})}+32 b \sum_{n=0}^{\infty} \frac{\exp \left[-(2 n+1)^{2} \pi^{2} \tau / 4\right]}{(2 n+1)^{2} \pi^{2}\left[(2 n+1)^{2} \pi^{2}-4 b\right]} .
$$

Note that $t_{\mathrm{d}}^{*}=t_{\mathrm{d}}\left(2 R T_{\mathrm{c}}\right)^{\frac{1}{2}} / a=2.00$ in the present calculation. As a result, $0.01 \leq b \leq 0.016$.

We now define the index of uniformity $\kappa(\tau, b)$ :

$$
\kappa(\tau, b)=1-\frac{T_{\mathrm{A}}^{*}(\tau ; b)}{T_{\mathrm{S}}^{*}(\tau ; b)} .
$$


$\kappa(\tau, b)$ provides a basis from which to discuss the legitimacy of the use of $T_{\mathrm{A}}$ for $T_{\mathrm{L}}$. We can secure the legitimacy if $\kappa(\tau, b)$ is sufficiently small. Figure A.10 shows the temporal change of $\kappa(\tau, b)$ for various $b$. Here, $\kappa(\tau, b)$ monotonously converges to zero as $\tau$ increases, regardless of the magnitude of $b$; hence, for any given $\nu$ such that $0 \leq \nu \leq 1$, there exists $\tau_{\nu}(b)$ that satisfies $\kappa\left(\tau_{\nu}, b\right)=\nu$. The smaller $b$ is, the larger $\tau_{\nu}(b)$ is, i.e., the slower $\kappa(\tau, b)$ converges to zero.

The temporal change of $\kappa^{0}(\tau)=\kappa(\tau, b \rightarrow 0)$, which is written as

$$
\kappa^{0}(\tau)=\lim _{b \rightarrow 0} \kappa(\tau, b)=1-\frac{1}{\tau}\left[\frac{1}{3}-32 \sum_{n=0}^{\infty} \frac{\exp \left[-(2 n+1)^{2} \pi^{2} \tau / 4\right]}{(2 n+1)^{4} \pi^{4}}\right],
$$

is also shown in Fig. A.10. The value of $\tau_{\nu}^{0}$ that satisfies $\kappa^{0}\left(\tau_{\nu}^{0}\right)=\nu$ provides the upper limit of $\tau_{\nu}(b)$. Note that $b$ obtained from the present calculation is small enough that $\tau_{\nu}(b)$ can be well approximated by $\tau_{\nu}^{0}$, as observed in Fig. A.10. The typical values of $\tau_{\nu}^{0}$ for $\nu=0.05$ and 0.02 are $\tau_{0.05}^{0}=6.67$ and $\tau_{0.02}^{0}=16.67$, respectively. Therefore, as far as $t>33.4\left(t_{\mathrm{d}}=2.00\right)$, We can regard the unsteady temperature field in the liquid 2 to be approximately spatially uniform (see the enlarged view of Fig. 4) because $\kappa(\tau, b)<0.02$ from the analytical solution of the one-dimensional heat conduction equation. The above investigation ensures that the heat transfer defined in the present study can be described by the one-dimensional heat conduction equation when there is no advection in liquid; that is, we can carry out the numerical simulation of vapor-liquid two-phase flow with unsteady net evaporation/condensation at a large spatial scale by coupling the one-dimensional heat conduction equation (Eq. (A.1)) and the Boltzmann equation, without carrying out the molecular simulation of the bulk liquid that contains a much larger number of molecules than those in the bulk vapor. 




Figure A.9: Fitting results of the temporal evolution of temperature of liquid 2.

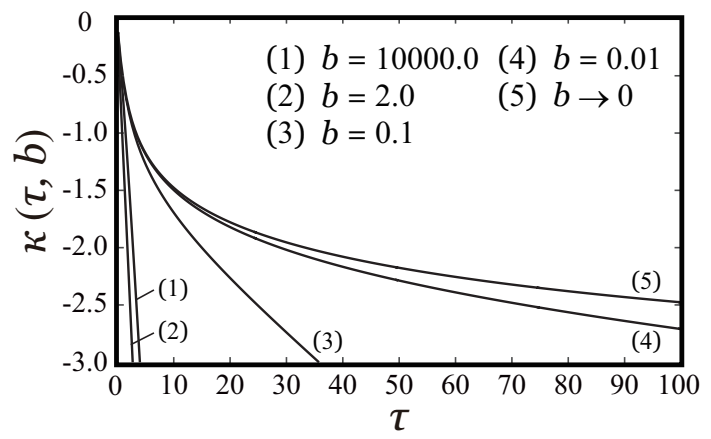

Figure A.10: Temporal change of the average temperature and required time. 


\section{References}

[1] J. Lee, T. Laoui, R. Karnik, Nanofluidic transport governed by the liquid/vapour interface, Nature Nanotechnology 9 (4) (2014) 317-323.

[2] S. Fujikawa, T. Akamatsu, Effects of the non-equilibrium condensation of vapour on the pressure wave produced by the collapse of a bubble in a liquid, Journal of Fluid Mechanics 97 (03) (1980) 481-512.

[3] I. Akhatov, O. Lindau, A. Topolnikov, R. Mettin, N. Vakhitova, W. Lauterborn, Collapse and rebound of a laser-induced cavitation bubble, Physics of Fluids 13 (10) (2001) 2805-2819.

[4] Y. Jinbo, T. Ogasawara, H. Takahira, Influence of the nonequilibrium phase transition on the collapse of inertia nonspherical bubbles in a compressible liquid, Experimental Thermal and Fluid Science 60 (2015) 374-384.

[5] J. G. Leidenfrost, On the fixation of water in diverse fire, International Journal of Heat and Mass Transfer 9 (11) (1966) 1153-1166.

[6] H. Linke, B. Alemán, L. Melling, M. Taormina, M. Francis, C. Dow-Hygelund, V. Narayanan, R. Taylor, A. Stout, Self-propelled leidenfrost droplets, Physical Review Letters 96 (15) (2006) 154502.

[7] D. Quéré, Leidenfrost dynamics, Annual Review of Fluid Mechanics 45 (2013) 197-215.

[8] C.-D. Ohl, M. Arora, R. Ikink, N. De Jong, M. Versluis, M. Delius, D. Lohse, Sonoporation from jetting cavitation bubbles, Biophysical journal 91 (11) (2006) 4285-4295.

[9] K. Kobayashi, T. Kodama, H. Takahira, Shock wave-bubble interaction near soft and rigid boundaries during lithotripsy: numerical analysis by the improved ghost fluid method, Physics in medicine and biology 56 (19) (2011) 6421.

[10] S. Sazhin, I. Shishkova, M. Al Qubeissi, A self-consistent kinetic model for droplet heating and evaporation, International Journal of Heat and Mass Transfer 93 (2016) 1206-1217.

[11] R. Meland, A. Frezzotti, T. Ytrehus, B. Hafskjold, Nonequilibrium molecular-dynamics simulation of net evaporation and net condensation, and evaluation of the gas-kinetic boundary condition at the interphase, Physics of Fluids 16 (2) (2004) 223-243.

[12] K. Gu, C. B. Watkins, J. Koplik, Molecular dynamics simulation of the equilibrium liquid-vapor interphase with solidification, Fluid Phase Equilibria 297 (1) (2010) 77-89.

[13] K. Gu, C. B. Watkins, J. Koplik, Multiscale molecular simulations of argon vapor condensation onto a cooled substrate with bulk flow, Physics of Fluids 22 (11) (2010) 112002.

[14] K. Kobayashi, K. Hori, M. Kon, K. Sasaki, M. Watanabe, Molecular dynamics study on evaporation and reflection of monatomic molecules to construct kinetic boundary condition in vapor-liquid equilibria, Heat and Mass Transfer (2015) 1-9.

[15] P. Barbante, A. Frezzotti, L. Gibelli, A kinetic theory description of liquid menisci at the microscale, Kinetic and Related Models 8 (2) (2015) 235-254.

[16] Y. Sone, Y. Onishi, Kinetic theory of evaporation and condensation-Hydrodynamic equation and slip boundary condition-, Journal of the Physical Society of Japan 44 (6) (1978) 1981-1994.

[17] Y.-P. Pao, Application of kinetic theory to the problem of evaporation and condensation, Physics of Fluids 14 (2) (1971) 306-312.

[18] T. Soga, A kinetic theory analysis of unsteady evaporation from a liquid surface with temperature change, Journal of the Physical Society of Japan 55 (5) (1986) 1556-1567.

[19] M. Matsumoto, Molecular dynamics of fluid phase change, Fluid Phase Equilibria 144 (1) (1998) 307314.

[20] T. Tsuruta, H. Tanaka, T. Masuoka, Condensation/evaporation coefficient and velocity distributions at liquid-vapor interface, International Journal of Heat and Mass Transfer 42 (22) (1999) 4107-4116.

[21] T. Ishiyama, T. Yano, S. Fujikawa, Molecular dynamics study of kinetic boundary condition at an interface between argon vapor and its condensed phase, Physics of Fluids 16 (8) (2004) 2899-2906.

[22] T. Ishiyama, T. Yano, S. Fujikawa, Kinetic boundary condition at a vapor-liquid interface, Physical Review Letters 95 (8) (2005) 084504.

[23] M. Bond, H. Struchtrup, Mean evaporation and condensation coefficients based on energy dependent condensation probability, Physical Review E 70 (6) (2004) 061605. 
[24] M. Kon, K. Kobayashi, M. Watanabe, Method of determining kinetic boundary conditions in net evaporation/condensation, Physics of Fluids 26 (7) (2014) 072003.

[25] M. Kon, K. Kobayashi, M. Watanabe, Numerical analysis of kinetic boundary conditions at net evaporation/condensation interfaces in various liquid temperatures based on mean-field kinetic theory, in: AIP Conference Proceedings, Vol. 1628, AIP Publishing, 2014, pp. 398-403.

[26] M. Kon, K. Kobayashi, K. Sasaki, M. Watanabe, Estimation of evaporation mass flux at vapor-liquid interface during nonequilibrium evaporation/condensation, Japanese Journal of Multiphase Flow: in japanese 29 (5) (2016) 493-500.

[27] M. Kon, K. Kobayashi, M. Watanabe, Liquid temperature dependence of kinetic boundary condition at vapor-liquid interface, International Journal of Heat and Mass Transfer 99 (2016) 317-326.

[28] A. Frezzotti, L. Gibelli, S. Lorenzani, Mean field kinetic theory description of evaporation of a fluid into vacuum, Physics of Fluids 17 (1) (2005) 012102.

[29] A. Frezzotti, M. Rossi, Slip effects at the vapor-liquid boundary, in: AIP Conference Proceedings, Vol. 1501, AIP Publishing, 2012, pp. 903-910.

[30] Y. Sone, Molecular gas dynamics: theory, techniques, and applications, Springer Science \& Business Media, 2007.

[31] S. Fujikawa, T. Yano, M. Watanabe, Vapor-liquid interfaces, bubbles and droplets: Fundamentals and applications, Springer Science \& Business Media, 2011.

[32] A. Kryukov, V. Y. Levashov, About evaporation-condensation coefficients on the vapor-liquid interface of high thermal conductivity matters, International Journal of Heat and Mass Transfer 54 (13) (2011) 3042-3048.

[33] M. Grmela, Kinetic equation approach to phase transitions, Journal of Statistical Physics 3 (3) (1971) $347-364$.

[34] J. Karkheck, G. Stell, Kinetic mean-field theories, The Journal of Chemical Physics 75 (3) (1981) $1475-1487$.

[35] N. F. Carnahan, K. E. Starling, Equation of state for nonattracting rigid spheres, The Journal of Chemical Physics 51 (2) (1969) 635-636.

[36] A. Frezzotti, A particle scheme for the numerical solution of the Enskog equation, Physics of Fluids 9 (5) (1997) 1329-1335.

[37] G. A. Bird, Molecular gas dynamics and the direct simulation of gas flows, Clarendon, 1994.

[38] K. Nanbu, Direct simulation scheme derived from the Boltzmann equation. I. Monocomponent gases, Journal of the Physical Society of Japan 49 (5) (1980) 2042-2049.

[39] T. Yano, Molecular dynamics study of nonequilibrium processes of evaporation and condensation at a vapor-liquid interface, in: AIP Conference Proceedings, Vol. 1501, 2012, p. 926. doi:10.1063/1.4769641.

[40] A. Frezzotti, P. Grosfils, S. Toxvaerd, Evidence of an inverted temperature gradient during evaporation/condensation of a Lennard-Jones fluid, Physics of Fluids 15 (10) (2003) 2837-2842.

[41] R. Meland, Molecular dynamics simulation of the inverted temperature gradient phenomenon, Physics of Fluids 15 (10) (2003) 3244-3247.

[42] R. Meland, T. Ytrehus, Dependence of the inverted temperature gradient phenomenon on the condensation coefficient, Physics of Fluids 16 (3) (2004) 836-838.

[43] P. Andries, P. L. Tallec, J.-P. Perlat, B. Perthame, The Gaussian-BGK model of Boltzmann equation with small prandtl number, European Journal of Mechanics - B/Fluids 19 (6) (2000) 813-830.

[44] C. Chu, Kinetic-theoretic description of shock wave formation. II., Physics of Fluids 8 (8) (1965) 14501455.

[45] A. Frezzotti, Boundary conditions at the vapor-liquid interface, Physics of Fluids 23 (3) (2011) 030609.

[46] K. Kobayashi, K. Ohashi, M. Watanabe, Numerical analysis of vapor-liquid two-phase system based on the Enskog-Vlasov equation, in: AIP Conference Proceedings, Vol. 1501, AIP Publishing, 2012, pp. $1145-1151$.

[47] H. S. Carslaw, J. C. Jaeger, Conduction of Heat in Solids, 2nd Edition, Oxford, 1959, pp. 92-104. 